\title{
Research on Applying Virtual Technology in Medical Informatization
}

\author{
Chi JinQing ${ }^{a}$, Hu Shengli ${ }^{b, ~}{ }^{*}$ and Guo Weic \\ Information center of Central Hospital of Cangzhou, Cangzhou, 061001, Hebei Province, China \\ ahushenglihsl@139.com, b404816467@qq.com, 642702380@qq.com, *Corresponding Author
}

\section{Keywords: Virtualization; Medicare; Informatization}

\begin{abstract}
Objectives: Promote the application of virtual technology in medical informatization, improve the resource utility ratio, reducing costs, and ensuring a secure and reliable system. Methods: Research the strengths, risks and limitations of virtual technology, based on the current application of virtual technology in medical informatization. Results: First, point out there are problems in wasting resources, high cost of maintenance, and the closed system; Second, illustrate the strengths of virtual technology such as enhancing the reliabilities of systems, the ability to recover from disasters, saving costs, and reducing the deployment cycles; Lastly, point out that the current virtual technology might not be applicable to all systems because of its own limitations, as well as the technical limitations and security risks in the virtual storage technique. Conclusions: Our research can serve as a guide in promoting the development, maturity and wide application of virtual technology.
\end{abstract}

\section{Introduction}

With the development of medical informatization, hospitals rely more and more on informatization in almost every area. And informatization also becomes increasingly professional and specific in recent years. In large top-tier hospitals, there typically exist various complex systems such as HIS, LIS, PACS and EMR. It has become a popular research area in medical informatization to manage all these complex systems in a scientific way, to improve utilization rate of the severs, to enhance the security of network and data, and to reduce costs so as to build a green hospital with the advanced technologies. In this paper, we present our research on the application of virtual technology in medical informatization, analyze the strengths/problems in medical informatization, and then give some suggestion on how to solve these problems along with pointing out the future work of virtual technology.

\section{Features and Categories of Virtualization}

Virtualization is the technique that allows computer applications to operate on virtual environment rather than operating on the real world. Virtual technology can enlarge the capacity of the hardware, simplify the configuration process of softwares, and improve the utilization rate of resources.

Based on the application field, virtualization technology can be divided into the following categories: server virtualization, desktop virtualization, storage virtualization, and network virtualization, etc. Further more the server virtualization can be divided into hardware virtualization, parallel virtualization, and operating system virtualization.

\section{Problems in Medical Informatization}

Resources Wasting. The Most of the systems in hospital are designed for specific applications, and each system varies in version, development platform and application. If a server is equipped with several systems, machines may break down or business may be interrupted due to the fact that the systems are not compatible with each other. If a server is matched with only one applied program, it will lead to limited storage of data, low utilization rate of the server, low load of the CPU, and vulnerable to single point failure. In addition, using more workstations and servers will consume 
more power and take up more space and the original server can't satisfy the existing demand, resulting in low utilization rate and serious resources waste.

High Costs of Maintenance. With the development of new business, we need to periodically increase the number of servers and clients. However, because of the special requirements of the hospital businesses, we need pay more and more for human maintenance in order to ensure an uninterrupted efficient operation of the business systems.

Closed System. To ensure the safety of the systems, hospitals often isolate internal networks from external ones. But in many cases external users need to visit the internal networks for online reservation and paper inquires. A closed system does not meet such requirement of medical informatization in the future.

Low Safety. Traditional application delivery modes and delivery framework have inherent problems. It is difficult to solve the fundamental problems such as data leaking and terminal threats. And the connection of external systems such as medical insurance and new rural cooperative medical care can bring some hidden threats to the information system of hospitals.

Low Utilization Rate of Storage. The storage of each system is unevenly distributed with low utilization rate. Virtual technology can centralize the data for collective storage.

\section{The Advantage of Applying Virtualization Technology in Medical Informatization}

Full Utilizing of the Available Resources. The use of virtualization technology in medical informatization has broken the common principle of "one server has one application program", and by integrating the physical server, the virtualization technology fully improves the server's processing capacity, increases the utilization rate of server resources and reduces the number of physical servers. Meanwhile, this technology uses light-weight client to reduce maintenance cost, energy consumption, and space usage, which is beneficial to reduce emission, and help maintain the Green IT.

Enhancement of Reliability. The virtualization technology is more tolerant to single point of failure. When a server breaks down, it can check itself and renew the system by modifying the hardware configuration. Besides, each virtual machine has its own copy of data , thus enhance the system reliability.

Recovery from System Failure. The virtual machine is stored in the external storage in the form of document, independent of the working hardware, thus separating the software and hardware. We can run the system and software in the virtual layer, independent of the specific hardware configuration in each backend. When there is failure, we can recover the system through fast transition and fast reproduction, avoiding labor-intensive manual recovery, thus enhance the operation's continuity [1,2].

Migration Online. The virtualization technology can complete online migration without disabling the service, maintain and update the server hardware and software, and also can achieve the moving of the entire data center [3,4]. The physical machine will be kept temporarily when moving, and it can be moved back if problems occur.

Dynamic Allocation of Resources. Hardware devices like CPU, diskette, internal storage and I/O can be stored in resource pools and managed dynamically though virtualization technology: it managers resources in the physical server and can assign the resource dynamically according to its load. Such technology enable one physical machine to be matched to several applications and those applications are separated from each other to ensure better safety and stability.

Enhancement of Network Integrity. By applying the light-weight client virtualization delivery model and architecture, we can build enough protection in the sharing network to ensure better safety and stability. This kind of network can support many kinds of services, such as voice, wireless, monitoring, internal and external network. It is an flexible technical solution, helping the service to be more open and integrated [5,6].

Increase Management Efficiency and Decrease Cost. The applying of virtualization technology in medical informatization can reduce power consumption, reduce the maintenance cost, and improve 
efficiency. Desktop virtualization is helpful for updating all workstations all together, integrating all system applications, decreasing maintenance volume, improving the security of data ,and achieving mobile working for medications [7].

Decrease the Deployment Cycle of the New Business. Informatization of new business requires to build a test platform. Traditional platform building is complicated. By applying virtual technology and selecting existing virtual machine snapshot templates to start the virtual machine, we can fastly copy, save, and manage the virtual machine and complete its testing in much shorter time.

\section{Analysis of the Limitation and Risk of Virtual Technology in Medical Information}

Analysis of Adaptability. Virtual technology has many advantages when applied to medical informatization. However, not all servers can be virtualized, since even the most advanced virtual technology could cause 5\%-10\% performance loss to the system. Systems with lots of graphs or data to write, such as PACS and LIS, requires high-speed and real-time data exchange, and requires better hardware and storage space. Such systems also requires large I/O operations and high throughput so they would consume more resources and affect the system performance. Besides, compared with physical machine, Virtualization platform is difficult to predict system failure and has limited service. If you start virtualization without recognizing the system load and resource utilization rate rules, you may have a unstable and high failure rate system. We should carefully analyze the specific situation before we want to apply virtual technology in Hospital. In particular, if the hardware does not meet the requirements, we should not use virtualization. For example, we should not virtualize non-essential applications or systems with low utilization rates [8-10]. On the other hand, for system like PACS which has large quantity of image transmission, it's better to establish cluster first and manage virtual machine on the cluster. All of its resources should be allocated by a centralized resource pool to achieve HA (high availability) and DRS (distributed resources scheduling) in the cluster. In this way we can guarantee load balance of multiple hosts and automatic recovery of fault hosts. Data should also be stored in one central disk array to improve the system performance. In practical, PACS virtualization has already been used in some hospital. Note that it is important to closely monitor the CPU and memory usage. If necessary, the space of CPU, memory and disk should be adjusted to ensure the reliability of the system. In addition, performance test should be done in advance to avoid significant performance decrease caused by large number of users.

Analysis of Limitation. By using Storage virtualization, we can achieve the free flow of data, improve storage performance, provide tolerant system, achieve multiple systems connection, and achieve integrated service. Meanwhile, we can avoid the loosely-coupled deployment and the independent apply pattern existing in the traditional storage technology, and make better use of the available resource. However, traditional virtual storage methods based on NAS and SAN have limitations when applied to systems with large number of cloud computing, high level application, strong extensible capacity, and high performance.

Analysis of Security. Systems are all centralized when applying virtual technology in Hospital. If one server is down then the entire hospital's service will be turned down and this would cause catastrophic effects. Virtualization technology itself has some security threats either: First, the traditional viruses, Trojans, and network attack remains a threat; Second, the virtual machine itself has security vulnerabilities; Third, patch updates may be delayed; Finally, the system vulnerability makes the hackers easy to attack. If we install the antivirus software in each virtual machine, the system performance would be significantly affected due to the limitation of hardware resources. Therefore, both virtualization platform and antivirus software developers need to be involved to build a secure virtualization platform. Meanwhile, if we install new applications on the Virtualization platform without extra hardware, it may reduce the security level of the system and cause the security hole in the configuration. The deployment and operation of virtual machine has become obstacles preventing us to apply virtual technology on the servers [11]. The raw virtual machine files are stored on the network, so the security of the virtual machine is highly dependent on the network storage. We should be careful when using the stored data replication function to backup the data, to best protect 
data security under existing conditions. At present, there are various virtualization technology on the market and various deployments policies. So the advantages/disadvantages and the security threats of different systems vary much. Rely solely on the security mechanism provided by the virtual technology itself is not sufficient to meet the security requirements. The virtualization deployment and configuration should be arranged carefully to cope with the growing complicated application environment [12, 13].

Analysis of Prospective. Existing virtualization technology mainly focus on the resource optimization in one single physical server. It lacks the ability to virtualize and optimize the global resources. With the developing of medical informatization, RHIS focusing on the resident's health record will become the focus of the area. And it needs the support from virtualization technology for visiting remote resources, building more flexible and reliable global resource space, establishing resources flow framework, achieving cross-physical machine resource optimization, and at the same time dynamically recognizing available resources. However, researches in such area are only in the test stage so far, and have not been largely deployed in practical applications [14].

Analysis of Standardization. The application of virtualization in the medical informatization is still in its early stage. Virtual technology and hardware developers do not have a uniformed standard, making some of the devices hard to use. To conclude, the standardization problems need to be solved.

\section{Summary}

The key technology adopted in regional medical is cloud computing and cloud storage. Virtualization technology can improve the level of resource utilization of the cloud storage system, enhance safety, eliminate data redundancy, and ensure the security of centralized data storage and business continuity. Although existing virtualization technology have some limitations in standardization and safety, with the continuing developing and improving of the virtualization technology it will play a tremendous role in constructing the IT infrastructure and promoting the development of medical informatization.

\section{References}

[1] Shi Lei, Luan ZhenTao. Oracle database migration based on Linux and virtual platform [J]. Chinese Medical Equipment Journal,38(2017) 69-71.

[2] Huang SiWen. Large Hospital Data Center Disaster Recovery Construction Based on Virtualization [J]. Journal of Medical Intelligence,38(2017) 40-42.

[3] PAN Chuandi. Building hospital local area network by virtual technology [J]. Chinese Hospitals,16(2012) 69-70.

[4] GONG Hai, CHEN Guo-qiang, XU Qiang,et al. Strengthening Hospital Network Security by Virtualization Technology [J]. Journal of Medical Intelligence,37(2016) 21-24.

[5] Zhang HaiBo, Zhou MinWei, Zhao XiaoQing. Application of Virtualization Technology in Medical Digital Resources Construction[J]. Chinese Journal of Health Informatics and Management,14(2017) 161-164.

[6] Sun XiaHuan. Construction of Hospital Virtual Data Center Based on VMware [J]. Chinese Journal of Health Informatics and Management,14(2017) 161-164.

[7] Zhu ZhuoJin, Zhao Xie, Li RuiYao et al. Reinforcing the Network System Infrastructure, Increasing the Broadband Operation Rate, to Prevent Security Risks [J]. Jiangsu Healthcare Administration,28(2017) 73-75.

[8] Su YueHong, Li Bin Shi, WenJuan et al. Informatization Construction and Transformation of Business System Hardware Platform in the Hospital [J]. Journal of Medical Intelligence,38(2017) 41-44. 
[9] Huang Wei, Liu Wei. Study on discipline construction in the transformation from clinical hospital to research hospital [J]. Chinese Hospitals,21(2017) 44-47.

[10]Zhou Yu, Wang WenMing, Liu Yun et al. Development and application of private cloud storage system [J]. Chinese Medical Equipment Journal,38(2017) 68-70.

[11] Wang ChunRong, Ceng YuPing. Research on the Construction of the Virtualized Cloud Platform for Hospitals [J]. Journal of Medical Intelligence,37(2016) 24-27.

[12]Tan YueQing, Hu JieTing. Journal of Clinical Psychosomatic Diseases [J]. Application of VMware vi rtualization technology in mental hospi tal,22(2016) 146-148.

[13] MENG Qun, QU Xiao-hui. Virtualization Technology Application in Integration of Hospital Information Platform Servers [J]. CHINA DIGITAL MEDICINE,6(2011) 8-12.

[14]LI Xian feng, WANG Kaiyun, LV Qing,et al. Research and practice of virtualization technology in third-grade ciass-A hospitals [J]. Chinese Hospitals,12(2012) 12-14. 